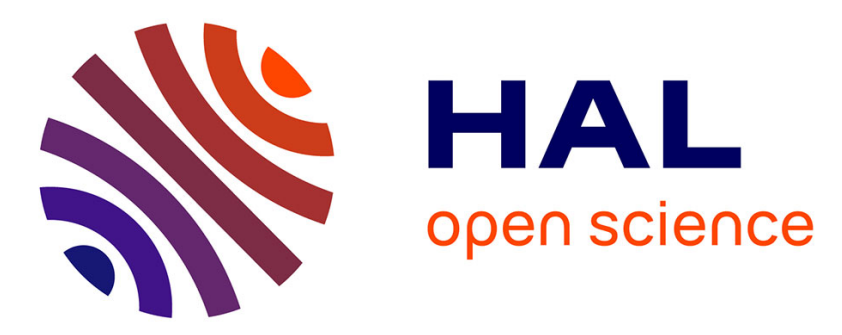

\title{
Interfacial magnetic domain wall formation in perpendicular-anisotropy, exchange-spring films
}

S.M. Watson, Thomas Hauet, J. A. Borchers, S Mangin, Eric E. Fullerton

\section{To cite this version:}

S.M. Watson, Thomas Hauet, J. A. Borchers, S Mangin, Eric E. Fullerton. Interfacial magnetic domain wall formation in perpendicular-anisotropy, exchange-spring films. Applied Physics Letters, 2008, 10.1063/1.2936836 . hal-01345239

\section{HAL Id: hal-01345239 \\ https://hal.science/hal-01345239}

Submitted on 13 Jul 2016

HAL is a multi-disciplinary open access archive for the deposit and dissemination of scientific research documents, whether they are published or not. The documents may come from teaching and research institutions in France or abroad, or from public or private research centers.
L'archive ouverte pluridisciplinaire HAL, est destinée au dépôt et à la diffusion de documents scientifiques de niveau recherche, publiés ou non, émanant des établissements d'enseignement et de recherche français ou étrangers, des laboratoires publics ou privés. 


\title{
Interfacial magnetic domain wall formation in perpendicular-anisotropy, exchange-spring films
}

\author{
S. M. Watson, ${ }^{1, a)}$ T. Hauet, ${ }^{2}$ J. A. Borchers, ${ }^{1}$ S. Mangin, ${ }^{3}$ and Eric E. Fullerton ${ }^{4}$ \\ ${ }_{1}^{1}$ NIST Center for Neutron Research, Gaithersburg, Maryland 20899-6102, USA \\ ${ }^{2}$ Hitachi Global Storage Technologies, San Jose, California 95135, USA \\ ${ }^{3}$ LPM, Nancy-Université, CNRS, Vandoeuvre-les-Nancy F-54506, France \\ ${ }^{4}$ Center for Magnetic Recording Research, University of California, San Diego, La Jolla, \\ California 92093-0401, USA
}

(Received 7 March 2008; accepted 5 May 2008; published online 22 May 2008)

\begin{abstract}
Using magnetometry and polarized neutron reflectometry (PNR), we have mapped the reversal processes of the antiferromagnetically coupled, hard/soft system, $\mathrm{TbFeCo} /\left[\mathrm{Co} / \mathrm{Pd}\left(t_{\mathrm{Pd}}\right)\right]_{15}$, with perpendicular anisotropy. The magnitude of the exchange coupling within the $[\mathrm{Co} / \mathrm{Pd}]$ layer can be tuned by varying Pd thickness. Since PNR is insensitive to moments perpendicular to the plane, a scattering geometry with polarization parallel to the scattering vector was used to isolate in-plane magnetization components and characterize the behavior of a domain wall near the bilayer interface. Consistent with predictions from a micromagnetic calculation, the characteristics of the domain wall vary with field and exchange stiffness in the Co/Pd. () 2008 American Institute of Physics.
\end{abstract}

[DOI: $10.1063 / 1.2936836$ ]

Today, perpendicular recording, in which the magnetic anisotropy axis is normal to the media film plane [perpendicular magnetic anisotropy (PMA)], has effectively replaced traditional in-plane storage media in the hard disk industry. ${ }^{1}$ Various exchange-coupled composite structures have been recently proposed to reduce the switching field amplitude of the media without reducing its thermal stability. ${ }^{1-3}$ In such soft/hard systems, the magnetization reversal is thought to occur by domain nucleation by the soft layer that propagates into the hard layer. Optimizing these structures requires controlling the vertical exchange and understanding the magnetic reversal pathway. To date, most of the experimental studies report macroscopic magnetization measurements or recording performances, ${ }^{1,4}$ providing only indirect information about the shape and the location of PMA domain walls (DWs). For related multilayer films with in-plane anisotropy, polarized neutron reflectivity (PNR) has been used in the past to resolve the depth dependence of interfacial magnetic DW. ${ }^{5,6}$ However, the application of this powerful technique to PMA media is nontrivial as the perpendicular moments are parallel to the scattering vector and do not give rise to scattering via the neutron selection rules. ${ }^{7}$

In this letter, we describe the depth-dependent, magnetic profile of a PMA exchange-coupled system obtained by using PNR in an unconventional geometry. ${ }^{8}$ Specifically, we have characterized antiferromagnetically (AF) coupled, $\mathrm{TbFeCo} /\left[\mathrm{Co} / \mathrm{Pd}\left(t_{\mathrm{Pd}}\right)\right]$ multilayers with varying Pd thickness, which could be part of a prototype for composite multilayer media. ${ }^{9}$ Room temperature PNR measurements, combined with magnetometry, directly reveal that an interfacial DW (iDW) nucleates as the field is cycled. A comparison between two samples with different $t_{\mathrm{Pd}}$ indicates that the internal exchange coupling of the soft layer influences the iDW shape and location during field cycles. The behavior of the iDW deduced from PNR studies is consistent with expectations

${ }^{a)}$ Electronic mail: shannon.watson@nist.gov. from an idealized one-dimensional (1D) micromagnetic calculation.

$\mathrm{Tb}_{45}\left(\mathrm{Fe}_{80} \mathrm{Co}_{20}\right)_{55} \quad(24.5 \mathrm{~nm}) /\left[\mathrm{Co}(0.35 \mathrm{~nm}) / \mathrm{Pd}\left(t_{\mathrm{Pd}}\right)\right]_{15} /$ $\operatorname{Pd}(2 \mathrm{~nm})$ multilayers with $t_{\mathrm{Pd}}=0.5$ or $0.7 \mathrm{~nm}$ were grown at room temperature onto $\mathrm{Si}$ wafers by dc magnetron sputtering. The ferrimagnetic TbFeCo alloy exhibits a strong perpendicular anisotropy and a net magnetization parallel to the $\mathrm{Tb}$ moments. ${ }^{10}[\mathrm{Co} / \mathrm{Pd}]$ multilayers have PMA for both samples, and the Pd thickness determines the exchange coupling between successive Co layers. ${ }^{11}$ The interfacial exchange interaction between the $[\mathrm{Co} / \mathrm{Pd}]$ and $\mathrm{TbFeCo}$ layers is dominated by the ferromagnetic exchange interaction between the transition metals such that AF coupling exists between the $\mathrm{TbFeCo}$ and $[\mathrm{Co} / \mathrm{Pd}]$ magnetizations.

Figure 1 shows the magnetization for $\mathrm{TbFeCo} /$ $\left[\mathrm{Co} / \mathrm{Pd}\left(t_{\mathrm{Pd}}\right)\right]$ samples with $t_{\mathrm{Pd}}=0.5 \mathrm{~nm}$ and $t_{\mathrm{Pd}}=0.7 \mathrm{~nm}$ measured as a function of field, applied perpendicular to the film plane. The two hysteresis curves are similar and made of three hysteretic regions as expected for an AF coupled

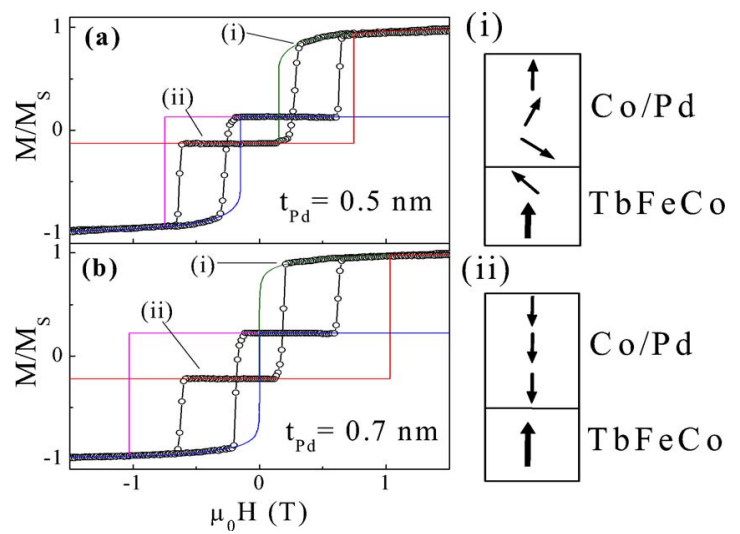

FIG. 1. (Color online) Magnetization vs field for films with Pd thicknesses of $0.5 \mathrm{~nm}$ (a) and $0.7 \mathrm{~nm}$ (b) taken at $300 \mathrm{~K}$. Black arrows indicate the direction of the field sweep. Colored lines correspond to model calculations. Cartoons illustrate the $\mathrm{Co}$ and the $\mathrm{TbFeCo}$ moments in large positive field (i) and zero field (ii). 
bilayer. ${ }^{6}$ In zero field, the $\mathrm{TbFeCo}$ and $\mathrm{Co} / \mathrm{Pd}$ layers are antiparallel [Fig. 1(ii)]. In applied fields, the interfacial exchange is overcome and a nonuniform iDW presumably forms [Fig. 1(i)]. For increasing field, the iDW is expected to compress against the interface with the TbFeCo.

We compare the normalized $M$ versus $H$ to the result of a 1D micromagnetic calculation based on a simplistic spin chain that mimics the bilayer (Fig. 1). The magnetic profile of this spin chain is characterized by a depth-dependent angle $\theta(z)$ relative to the chain axis, as described elsewhere. ${ }^{12,13}$ Exchange, anisotropy, and Zeeman energies are minimized numerically to determine the (meta)stable magnetic configurations, which correspond to minima of the total magnetic energy (colored lines in Fig. 1) at each field value. Magnetization measurements compare well to the calculations for both samples [Figs. 1(a) and 1(b)] using the magnetization values ${ }^{14} M_{\mathrm{TbFeCo}}=330 \mathrm{kA} / \mathrm{m}$, $M_{\mathrm{Co}(0.35) / \operatorname{Pd}(0.5)}=802 \mathrm{kA} / \mathrm{m}, M_{\mathrm{Co}(0.35) / \operatorname{Pd}(0.7)}=770 \mathrm{kA} / \mathrm{m}$, the anisotropy (which includes magnetocrystalline and shape anisotropy) constants $\quad K_{\mathrm{TbFeCo}}=3.3 \times 10^{7} \mathrm{erg} \mathrm{cm}^{-3}$, $K_{\mathrm{Co}(0.35) / \operatorname{Pd}(0.5)}=2.5 \times 10^{7} \mathrm{erg} \mathrm{cm}^{-3}, \quad K_{\mathrm{Co}(0.35) / \operatorname{Pd}(0.7)}=3.5$ $\times 10^{7} \mathrm{erg} \mathrm{cm}^{-3}$, and the exchange stiffness: $A_{\mathrm{TbFeCo}}$ $=10^{-6} \mathrm{erg} \mathrm{cm}, \quad A_{\mathrm{Co}(0.35) / \operatorname{Pd}(0.5)}=5 \times 10^{-7} \mathrm{erg} \mathrm{cm}, \quad$ and $A_{\mathrm{Co}(0.35) / \mathrm{Pd}(0.7)}=1 \times 10^{-7} \mathrm{erg} \mathrm{cm}$. The interfacial exchange coupling is held constant at $A_{\text {int }}=-9 \mathrm{erg} \mathrm{\textrm {cm } ^ { 2 }}$.

PNR measurements were made on the NG1 reflectometer at NIST to determine the shape and extent of the DW in the $\mathrm{TbFeCo} /[\mathrm{Co} / \mathrm{Pd}]$ samples. In most typical PNR experiments, the neutron polarization and applied field are both oriented within the sample plane. In our study, we instead aligned both the neutron polarization and external field perpendicular to the sample plane, ${ }^{8}$ parallel to the direction of the scattering vector, in order to isolate the in-plane moment projection. We measured all four cross sections: $R^{+-}$and $R^{--}$ labeled nonspin flip (NSF) as the neutron retains its original polarization, and $R^{+-}$and $R^{-+}$, labeled spin flip (SF), where the neutron spin rotates $180^{\circ}$. In this scattering geometry, the NSF reflectivity has no magnetic component and is sensitive only to the chemical depth profile of the film. The SF reflectivity originates entirely from the projection of the moment in the film plane because the moment parallel to $Q$ does not contribute to any of the scattering in accordance with neutron selection rules. ${ }^{15}$ Since our samples have PMA, SF scattering develops only when an iDW is formed in an applied field. The depth dependence of the in-plane moment projection, extracted from the SF data, can thus be used to deduce the shape and position of the DW in the multilayer.

Figure 2 shows SF reflectivity data (i.e., the sum of $R^{+-}$ and $R^{-+}$, which are equal ${ }^{16}$ ) for the film with nominal Pd thickness of $0.5 \mathrm{~nm}$ measured at different fields after positive saturation. An increase in the SF intensity is observed with decreasing field from 0.7 to $0.3 \mathrm{~T}$. Since the SF scattering amplitude is directly related to the component of the magnetization in the film plane, this observation corroborates the DW decompression scenario described above. The SF amplitude is much smaller at $0.005 \mathrm{~T}$, however, consistent with formation of an antiparallel state with the moments parallel to the anisotropy axis. A similar behavior was observed in the PNR data for the $\mathrm{Pd}=0.7 \mathrm{~nm}$ film.

To obtain the chemical and magnetic depth profiles, the PNR data were fitted to the theoretical formalism described elsewhere ${ }^{17}$ with the REFLPAK software ${ }^{18}$ Prior to fitting, the

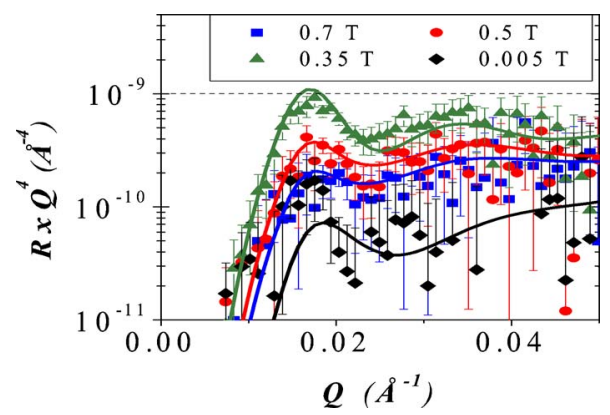

FIG. 2. (Color online) Comparison of the SF reflectivity data for the film with Pd thickness of $0.5 \mathrm{~nm}$ (left). Solid lines are fits to the data. The SF reflectivity at intermediate fields from the in-plane projection of the moment is consistent with DW formation.

reflectivity data were corrected for background, efficiencies of the polarizing elements (typically $>97 \%$ ), and footprint of the beam. To reduce the number of variables, key structural parameters, obtained from fits to the x-ray reflectivity data, were held constant during fits to the NSF neutron data. The SF data (Fig. 2) were then fitted by varying the in-plane projection of the magnetization $M_{\text {in }}(z)$ while requiring a smooth, continuous variation with depth. The data are well described by a model in which the Co moment gradually increases with depth [Fig. 3(a)]. Alternate magnetic structures involving, for example, uniform alignment of all inplane moments or a gradual decrease in the Co moments with increasing depth could not account for prominent data features, such as the width and depth of the dip near $Q=0.025 \AA^{-1}$. Overall, the fits are much less sensitive to the characteristics of the $\mathrm{TbFeCo}$ moment.

Figure 3 summarizes the depth-dependent, in-plane component of the magnetization deduced from fits to the SF reflectivity for our films with $t_{\mathrm{Pd}}=0.5 \mathrm{~nm}$ [Fig. 3(a)] and $t_{\mathrm{Pd}}=0.7 \mathrm{~nm}$ [Fig. 3(b)], in comparison to $1 \mathrm{D}$ micromagnetic calculations [Figs. 3(c) and 3(d), respectively] that fit the magnetization curves. For all of the fields except $0.005 \mathrm{~T}$, the fits reveal that a large fraction of the moments are not aligned parallel to the PMA axis. Starting at the top of the $\mathrm{Co} / \mathrm{Pd}$ stack (i.e., depth of zero), the in-plane magnetization

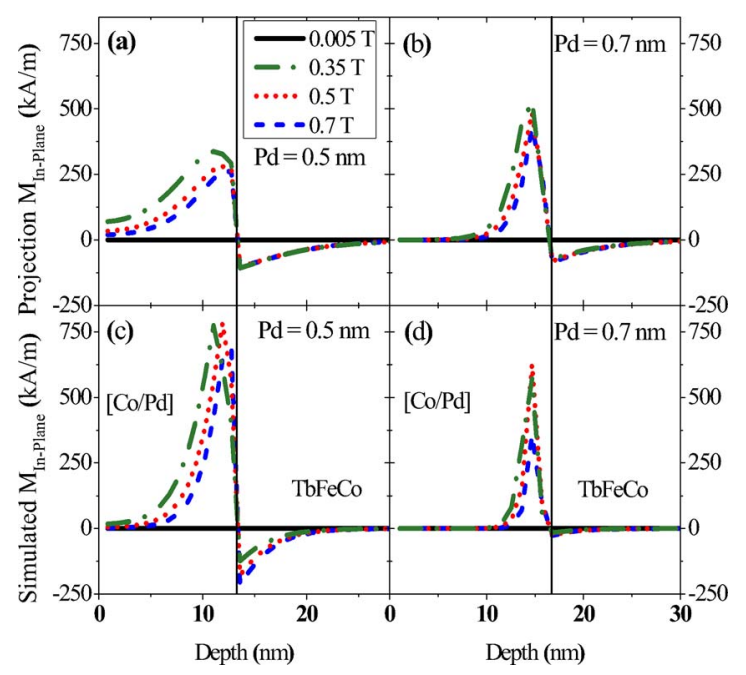

FIG. 3. (Color online) In-plane magnetization as a function of depth for various applied fields. (a) and (b) show the fit to the PNR data. (c) and (d) show the 1D simulation. The black vertical line indicates the boundary between the $\mathrm{Co} / \mathrm{Pd}$ superlattice and the $\mathrm{TbFeCo}$ layer.

AIP license or copyright; see http://apl.aip.org/apl/copyright.jsp 
projection increases until it reaches a maximum close to the $[\mathrm{Co} / \mathrm{Pd}]-\mathrm{TbFeCo}$ interface. The magnetization then decreases steeply and reverses direction within the $\mathrm{TbFeCo}$ layer as a result of the AF interface coupling. This depth dependence mimics that of the in-plane moment projection of a "Neel-like" iDW predicted by the 1D model. This iDW is localized mainly in the $[\mathrm{Co} / \mathrm{Pd}]$ multilayer. An estimate for the DW thickness is $\delta=\pi \sqrt{A / K}$. Using the magnetic parameters above, the calculated DW thickness is about $4.5 \mathrm{~nm}$ for $t_{\mathrm{Pd}}=0.5 \mathrm{~nm}$ and about $1.5 \mathrm{~nm}$ for $t_{\mathrm{Pd}}=0.7 \mathrm{~nm}$. The latter is much smaller as a result of the smaller exchange coupling across the $\mathrm{Pd}$ layers. As the field is increased from 0.35 to $0.7 \mathrm{~T}$, the total amplitude of the in-plane magnetization decreases, and the iDW center (i.e., the depth at which the in-plane magnetization is maximum) slightly shifts toward the interface.

The extent and position of the iDW in both samples qualitatively match the model calculations, confirming that the $\mathrm{Pd}$ thickness dependence originates from differences in the $\mathrm{Co}-\mathrm{Co}$ interlayer exchange coupling in the $\mathrm{Co} / \mathrm{Pd}$ multilayer. The in-plane moment magnitude of the $0.7 \mathrm{~nm}$ sample is close to model predictions, but the in-plane moment for the $0.5 \mathrm{~nm}$ sample is smaller than expected (Fig. 3). This discrepancy ${ }^{19}$ could arise from a distribution of iDWs across the sample plane with small variations in $\delta$. This distribution would reduce the net in-plane layer moment measured by PNR. In addition, the $1 \mathrm{D}$ model restricts rotation of the spins to a single plane. While our PNR results are consistent with a Neel-like wall, they do not exclude the possibility of more complex DWs with in-plane and out-of-plane components.

In conclusion, we have studied $\mathrm{TbFeCo} /[\mathrm{Co} / \mathrm{Pd}]$ bilayers with an AF interface exchange coupling and uniaxial perpendicular anisotropy (PMA). Our PNR and magnetometry measurements directly reveal that an iDW forms at intermediate fields due to competition between Zeeman and exchange coupling energies. Our PNR scattering geometry was optimized to isolate the in-plane footprint of the iDW, since PNR is not directly sensitive to moment components perpendicular to the sample plane. Our results reveal that the shape and location of the iDW vary systematically with the exchange stiffness of the $\mathrm{Co}-\mathrm{Co}$ interaction of the $\mathrm{Co} / \mathrm{Pd}$ bi- layers and with applied field. The magnetic configuration obtained from PNR also compares favorably to predictions from an idealized spin-chain model. These results highlight the strong Co-Co interaction dependence on the thickness of the Pd interlayers. (A $0.2 \mathrm{~nm}$ change in Pd thickness results in a factor of 5 change in the exchange coupling with a concomitant change in domain profile.) Our results thus open the door for direct characterization of the depth-dependent magnetic structure in composite PMA recording media where quantitative understanding of interlayer coupling and incoherent reversal is the key.

${ }^{1}$ H. J. Richter, J. Phys. D: Appl. Phys. 40, R149 (2007); N. Supper, D. T. Margulies, A. Moser, A. Berger, H. Do, and E. E. Fullerton, IEEE Trans. Magn. 41, 3238 (2005).

${ }^{2}$ D. Suess, T. Schrefl, S. Fahler, M. Kirschner, G. Hrkac, F. Dorfbauer, and J. Fidler, Appl. Phys. Lett. 87, 012504 (2005).

${ }^{3}$ A. Yu. Dobin and H. J. Richter, Appl. Phys. Lett. 89, 062512 (2006).

${ }^{4}$ J. P. Wang, W. K. Shen, J. M. Bai, R. H. Victora, J. H. Judy, and W. L. Song, Appl. Phys. Lett. 86, 142504 (2005).

${ }^{5}$ M. F. Toney, J. A. Borchers, K. V. O'Donovan, C. F. Majkrzak, D. T. Margulies, and E. E. Fullerton, Appl. Phys. Lett. 86, 162506 (2005).

${ }^{6}$ T. Hauet, J. A. Borchers, Ph. Mangin, Y. Henry, and S. Mangin, Phys. Rev. Lett. 96, 067207 (2006).

${ }^{7}$ G. L. Squires, Contemp. Phys. 17, 411 (1976).

${ }^{8}$ W. Lohstroh, M. Münzenberg, W. Felsch, H. Fritzsche, H. Maletta, and G. P. Felcher, J. Magn. Magn. Mater. 198, 440 (1999).

${ }^{9}$ S. Hernandez, M. Kapoor, and R. H. Victora, Appl. Phys. Lett. 90, 132505 (2007)

${ }^{10}$ M. H. Kryder, J. Appl. Phys. 57, 3913 (1985).

${ }^{11}$ E. E. Fullerton, D. Stoeffler, K. Ounadjela, B. Heinrich, Z. Celinski, and J. A. C. Bland, Phys. Rev. B 51, 6364 (1995).

${ }^{12}$ F. Montaigne, S. Mangin, and Y. Henry, Phys. Rev. B 67, 144412 (2003).

${ }^{13}$ E. E. Fullerton, J. S. Jiang, M. Grimsditch, C. H. Sowers, and S. D. Bader, Phys. Rev. B 58, 12193 (1998).

${ }^{14}$ The values for $M_{\mathrm{Co}(3.5) / \mathrm{Pd}(X)}$ are normalized relative to the total $\mathrm{Co} / \mathrm{Pd}$ multilayer thickness.

${ }^{15}$ R. M. Moon, T. Riste, and W. C. Koehler, Phys. Rev. 181, 920 (1969).

${ }^{16} \mathrm{~A}$ difference between $R^{+-}$and $R^{-+}$would be indicative of a spiral domain wall with a preferred chirality (see Ref. 8).

${ }^{17}$ C. F. Majkrzak, Physica B 221, 342 (1996).

${ }^{18}$ P. A. Kienzle, K. V. O’Donovan, J. F. Ankner, N. F. Berk, and C. F. Majkrzak, http://www.ncnr.nist.gov/reflpak(2000-2006).

${ }^{19}$ The effective Zeeman splitting of the SF intensity was negligible and cannot account for the observed moment reduction, with the exception of the low- $Q, 0.7$ T data; see G. P. Felcher, S. Adenwalla, V. O. De Haan, and A. A. Van Well, Nature (London) 377, 409 (1995). 